\title{
Ab Initio Studies of Electromechanical Effects in Carbon Nanotubes
}

\author{
M. Verissimo-Alves, ${ }^{1}$ R.B. Capaz, ${ }^{1}$ Belita Koiller, ${ }^{1}$ Emilio Artacho, ${ }^{2}$ and H.Chacham ${ }^{3}$ \\ ${ }^{1}$ Instituto de Física, Universidade Federal do Rio de Janeiro, \\ Caixa Postal 68528, Rio de Janeiro, RJ, Brazil, 21945-970, \\ ${ }^{2}$ Departamento de Física de la Matéria Condensada, C-II and Instituto Nicolás Cabrera, \\ Universidad Autónoma de Madrid, 28049, Madrid, Spain \\ ${ }^{2}$ Departamento de Física, ICEx, Universidade Federal de Minas Gerais, Caixa Postal 702, \\ 30123-970 Belo Horizonte, MG, Brazil
}

Received on 23 April, 2001

\begin{abstract}
Carbon nanotubes have recently attracted interest for their possible applications as nanoactuators and nanoswitches, as well as possible building blocks for nanoelectronics. We present ab initio calculations for uniformly charged graphene and $(11,0),(9,0)$ and $(5,5)$ carbon nanotubes. We also consider the effects of polaron formation in these systems. The strain-charge coefficient is calculated and compared for both graphene and the nanotubes under study.
\end{abstract}

\section{Introduction}

Carbon nanotubes are recently discovered materials, whose mechanical and electronic properties have attracted a lot of attention from both fundamental and applied research. Their unique properties enable one to study one-dimensional electronic systems (experimentally and theoretically) and several devices and applications have been envisaged using them as fundamental blocks.

One of these recently realized applications of carbon nanotubes consists in an artificial "muscle" [1], which is composed of two sheets of nanotube paper glued to a double-face Scotch tape. These sheets are then immersed in an electrolytic solution and a low potential difference is applied to both sides of the sheet. With the application of the voltage, it is observed that the sheet contracts appreciably, in the order of a few $\mathrm{mm}$. All the properties measured from this raw prototype indicate that it is a promising candidate for a switching nanodevice.

In this work, we consider electromechanical effects in carbon nanotubes which are probably related to the experimental observations [1]. In the first part, we present results on uniform charging of graphene sheets and semiconducting and metallic carbon nanotubes, comparing calculated and experimental strain-charge coefficients. In the second part, we discuss the implications of self charge-localization in the form of polarons to the observed electromechanical effects [1] and also to recently observed opto-mechanical effects [2].

\section{Uniformly Charged Graphene and Nanotubes}

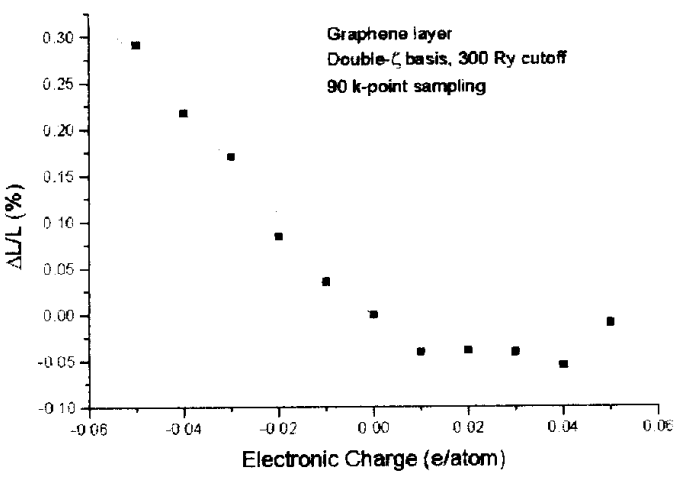

Figure 1. $\Delta L / L$ versus electronic charge for a graphene sheet.

We start by presenting results obtained through $a b$ initio calculations for uniformly charged graphene layers, semiconducting and metallic carbon nanotubes. The problem of charged graphitic materials is of great technological interest due to the possibility of making batteries with greater charge storage capacity. For instance, intercalation of alkali atoms between graphene layers drives charge transfer from the former to the later. Previous theoretical studies [3] indicate that the presence of these donor ions, besides inducing charge transfer to graphene, also causes electronic tranfer from 
$\sigma$ to $\pi$ orbitals within the carbon network, therefore increasing the lattice parameter of graphite. An opposite effect (contraction) occurs for acceptor ions. Therefore this effect is of direct interest to the studies on artificial "muscles", since the authors in [1] propose that charge transfer from the electrolytic solution is the key to the observed electromechanical actuation.

Our calculations are performed using the SIESTA[4] code, a numerical-atomic-orbital method based on density functional theory. This technique has been successfully applied to a number of studies involving nanotubes $[5,6]$. We use a generalized gradient approximation for exchange and correlation [7] and norm conserving pseudopotentials [8]. A split-valence double- $\zeta$ basis of pseudoatomic orbitals with an orbital confining energy of 0.3 $\mathrm{eV}$ and an energy cutoff of $300 \mathrm{Ry}$ for the fast Fourier transform integration mesh are used. Extra electronic charge is introduced in the supercell and it is compensated by a uniform background ("jellium").

Results for $\Delta L / L$ vs. $\Delta q$ ) are presented in Figs. 1 and 2. For graphene (Fig. 1) and metallic nanotubes (Figs. 2(a) and 2(b)) we notice a linear behavior from large negative charges (donors) to small positive charges (acceptors) and a saturation for large positive charges. The strain-charge coefficients, defined by $C_{s c}=\Delta L /(L \Delta q)$, are extracted from the linear portion of the graphs, as indicated by the linear fit. For semiconducting $(11,0)$ nanotubes (Fig. 2(c)) we notice a striking difference in the electromechanical response: An increase in the lattice constant is observed for both positive and negative charges. This is probably due to the effect of occupying anti-bonding states (negative extra charge) and unoccupying bonding states (positive extra charge).

Calculated $C_{s c}$ are shown in Table I. The agreement with experimental results is excellent for graphene. Also, we notice that $C_{s c}$ values are larger for carbon nanotubes in comparison with graphene, as noticed in experiment.
Table I. Strain-charge coefficients for graphene and

\begin{tabular}{ccc}
\multicolumn{3}{c}{ carbon nanotubes } \\
\hline Material & $C_{s c}$ & Experimental value \\
\hline graphene & 0.059 & 0.066 \\
$(5,5)$ nanotube & 0.09 & 0.17 (sheet) \\
$(9,0)$ nanotube & 0.10 & 0.17 (sheet) \\
$(11,0)$ nanotube* & 0.12 & 0.17 (sheet) \\
\hline
\end{tabular}
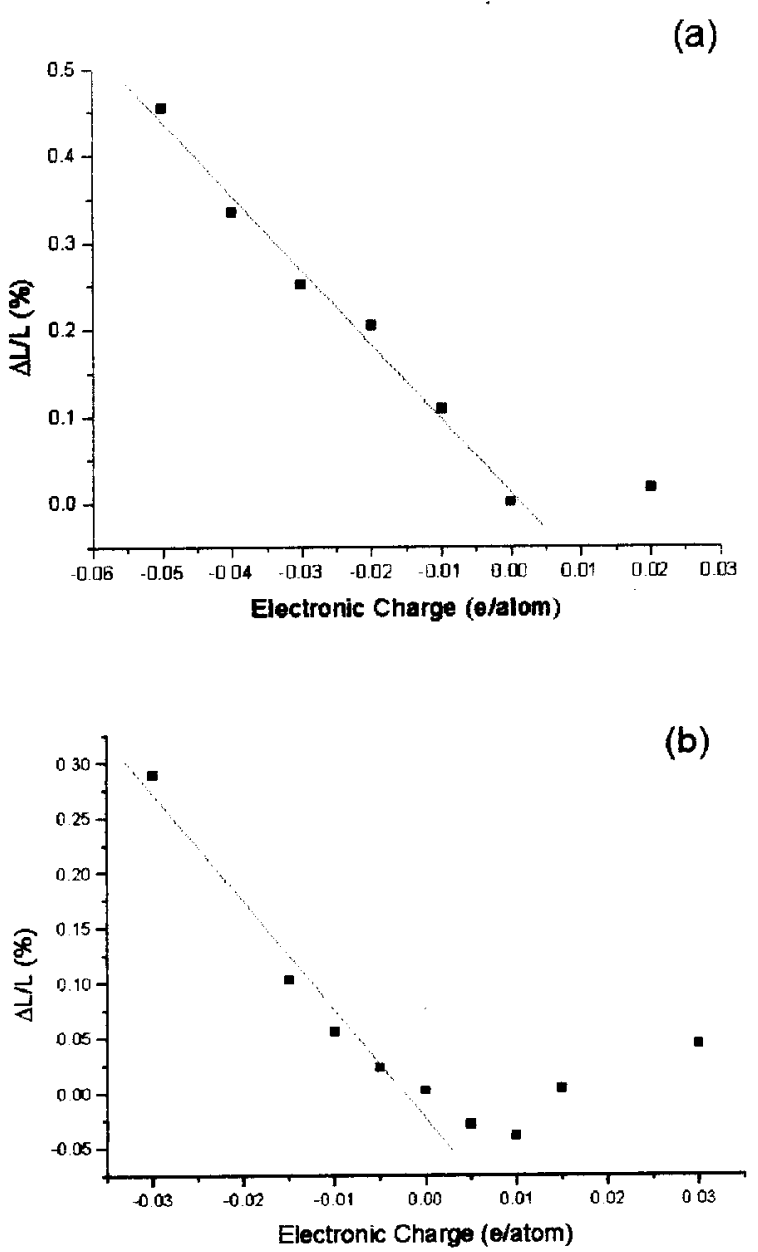

(c)

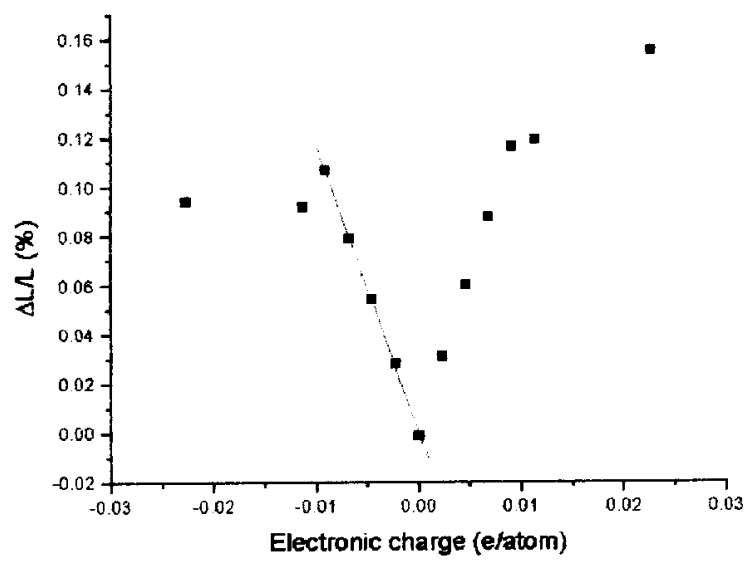

Figure 2. $\Delta L / L$ versus electronic charge for (a) $(9,0)$, (b) $(5,5)$ and (c) $(11,0)$ carbon nanotubes.

\section{Polarons in Carbon Nan- otubes}

Now we discuss the possibility of polaronic formation in carbon nanotubes and its possible relationship to the 
electromechanical and opto-mechanical effects observed recently $[1,2]$. Taking a neutral semiconducting nanotube with its axis lying along the $z$ direction, we take into acount two types of distortions in the nanotube lattice: a radial distortion and an axial distortion, characterized by the strain parameters $\epsilon_{r}=\left(R-R_{0}\right) / R_{0}$ and $\epsilon_{z}=\left(\ell-\ell_{0}\right) / \ell_{0}$. The hamiltonian for the system is written, in a continuum approximation as

$$
\begin{aligned}
E & =-\frac{\hbar^{2}}{2 m_{e f f}} \int_{-\infty}^{+\infty} \psi^{*}(z) \frac{d^{2}}{d z^{2}} \psi(z) d z \\
& +\lambda_{r} \int_{-\infty}^{+\infty} \psi^{*}(z) \psi(z) \epsilon_{r}(z) d z \\
& +\lambda_{z} \int_{-\infty}^{+\infty} \psi^{*}(z) \psi(z) \epsilon_{z}(z) d z \\
& +\frac{k_{r}}{2} \int_{-\infty}^{+\infty} \epsilon_{r}^{2}(z) d z+\frac{k_{z}}{2} \int_{-\infty}^{+\infty} \epsilon_{z}^{2}(z) d z \\
& +k_{r z} \int_{-\infty}^{+\infty} \epsilon_{r}(z) \epsilon_{z}(z) d z
\end{aligned}
$$

where $k_{r, z, r z}$ are the spring constants for radial, axial and radial-axial distortions and $\lambda_{r, z}$ are the electronphonon coupling constants rela tive to axial and radial distortion, respectively. The minimization of (1) with respect to $\epsilon_{r, z}$ yields a nonlinear Schrödinger equation of the form

$$
\left[\frac{d^{2}}{d z^{2}}-\tilde{C} \psi^{*}(z) \psi(z)\right] \psi(z)=\tilde{\varepsilon} \psi(z),
$$

where the $C$ 's are constants that depend on the $k$ 's and $\lambda$ 's. Solving (2) we find the shape for the polaron, its effective mass (in a semiclassical approximation) and typical length for both electrons and holes with the $k$ 's and $\lambda$ 's found from $a b$ initio calculations for $(7,0)$ and $(11,0)$ nanotubes. The details of the analytical and numerical calculations can be found in [9]. Here we review only the main results and possible implications for electromechanical and opto-mechanical effects reported recently $[1,2]$.

Our results yield rather delocalized polarons with the typical length being of the order of $200 \AA$ for a hole in a carbon nanotube. Therefore, a $(7,0)$ nanotube would have enough room for 500 hole polarons, which would cause a change in length of about $10 \AA$, sensible enough to be measured by STM. We also calculate the strain-charge coefficients. We find that the calculated value, $C_{s c}=0.15$, is in the range of the experimental value, $C_{s c}=0.17$, indicating that polaron formation may have an important role in the observed electromechanical effect. We also notice that the calculation of $C_{s c}$ for the case where we consider charge self-localization may help to enhance the value calculated for the case of uniformly charged nanotubes.

We also find that different carriers, in the same nanotube, may have different effects on the axial lattice distortion. We found that both an electron and a hole in an $(11,0)$ nanotube cause an axial expansion, whereas in the $(7,0)$ nanotube, a hole causes a contraction, and an electron causes an axial expansion. Therefore, we can predict strong and weak responses to light depending on the type of nanotube. An (11,0)-like nanotube would have a strong response to light, since the effects are alike and reinforce each other. On the other hand, a $(7,0)$-like nanotube would have a weaker response to light, since the effects are opposite and may partially or totally cancel each other.

We ackowledge financial support from Brazilian agencies FAPERJ, CNPq, CAPES, PRONEX-MCT and FUJB-UFRJ.

\section{References}

[1] R. H. Baughman, C. Cui, A. A. Zakhidov, Z. Iqbal, J. N. Barisci, G. M. Spinks, G. G. Wallace, A. Mazzoldi, D. De Rossi, A. G. Rinzler, O. Jaschinski, S. Roth, and M. Kertesz, Science 284, 1340 (1999).

[2] Y. Zhang and S. Iijima, Phys. Rev. Lett. 82, 3472 (1999).

[3] C. T. Chan, W. A. Kamitakahara, K. M. Ho, P. C. Eklund, Phys. Rev. Lett., 58, 1528 (1987).

[4] D. Sánchez-Portal, P.Ordejón, E. Artacho and J. M. Soler, Int. J. Quantum Chem. 65, 453 (1997); P.Ordejón, E. Artacho and J. M. Soler, Phys. Rev. B 53, R10441 (1996).

[5] M. S. C. Mazzoni and H. Chacham, Appl. Phys. Lett. 76, 1561 (2000).

[6] D. Sánchez-Portal, E. Artacho, J. M. Soler, A. Rubio and P. Ordejon, Phys. Rev. B 5912678 (1999).

[7] J. P. Perdew, K. Burke, and M. Ernzerhof, Phys. Rev. Lett. 77, 3865 (1996).

[8] N. Troullier and J. L. Martins, Phys. Rev. B 43, 2006 (1991).

[9] M. Verissimo-Alves, R. B. Capaz, Belita Koiller, Emilio Artacho and H. Chacham, Phys. Rev. Lett 86, 3372 (2001). 